\title{
Designing the ultrasonic system used in industrial air filtration based on the ultrasonic cavitation phenomenon
}

\author{
Dan Niţoi ${ }^{1, *}$, Zoia Apostolescu ${ }^{1}$, Constantin Petriceanu ${ }^{1}$, and Corneliu Rontescu ${ }^{1}$ \\ ${ }^{1}$ University Politehnica of Bucharest, Splaiul Independenţei no. 313, Bucharest, Romania
}

\begin{abstract}
The paper presents the activity developed developed in the field of polluted air purification from industrial shop (paint shops, foundries, welding constructions, forging, etc.). The advantages of using ultrasounds in the pollutant air filtration and purification process and an ultrasonic filter designed are presented considering the ultrasonic cavitation phenomenon. The main computational and construction elements of the ultra-acoustic system used in the design of the air filter are presented in the paper. The work focuses in the design of the main part of the ultrasonic system represented by ultrasonic transducer. In this case, the transducer network that is used in ultrasonic cavitation method consists of ten ultrasonic transducers. Each one of them consists of asymmetrical passive elements (reflector and radiant element).The design steps and the use of FEM as working method will provide the theoretical basis of the operation of the ultrasonic system so that it allows air filtration based on the ultrasonic acoustic cavitation phenomenon.
\end{abstract}

\section{Introduction}

Because the main phenomenon used in the ultrasonic filtration process is ultra-acoustic cavitation, a phenomenon that occurs at the propagation of ultrasonic waves through a liquid medium, the team has carried out a series of researches on ultra-acoustic cavitation, some of them, those with applicability in the air filtering, is presented below. According to modern hydrodynamic theory there is a complete analogy between liquids and gases in that in both environments can propagate only longitudinal waves. Considering the viscosity of the liquid due to which both a compressive stress and a cutting stress can be applied to it, then the liquid state can be considered closer to the solid state than the gaseous state and therefore transversal wave can be considered.

In a viscoelastic environment in the static state, between the compression part due to the force $\mathrm{p}$ with the deformation $\mathrm{s}$ and the cutting part due to the $\mathrm{p}_{\mathrm{ij}}$ effort, with the deformation of the $\mathrm{j}_{\mathrm{ig}}$, the following relations are available:

\footnotetext{
* Corresponding author: nitoidan@yahoo.com
} 


$$
p=-k_{0} s ; \quad p_{i j}=G \cdot s_{i j}
$$

When a variable pressure is applied to the fluid, the compression module $\mathrm{K}$ becomes a function of the static compression modulus $\mathrm{K}_{0}$ and the rotational modulus $\mathrm{K}_{1}$ that occurs in the relaxation process, i.e.:

$$
\begin{gathered}
K=K_{0}+K_{1} \frac{\omega^{2} \tau_{v}^{2}}{1+\omega^{2} \tau_{v}^{2}}+j k_{1} \frac{\omega \tau_{v}}{1+\omega^{2} \tau_{v}^{2}} \\
K_{1}=\frac{\eta_{v}}{\tau_{v}}
\end{gathered}
$$

Wherein: $\tau_{v}$ is the relaxation time under constant volume; $\eta_{v}$ - volume viscosity.

Analogously, under the action of an alternate effort, the cutting module $\mathrm{G}$ becomes an expression of the form:

$$
\begin{gathered}
G=G_{1} \frac{\omega^{2} \tau_{s}^{2}}{1+\omega^{2} \tau_{s}^{2}}+j G_{1} \frac{\omega \tau_{s}}{1+\omega^{2} \tau_{s}^{2}} \\
G_{1}=\frac{\eta}{\tau_{s}}
\end{gathered}
$$

in which: $\tau_{s}$ - it is the time of relaxation; $\eta$-viscosity.

\subsection{Ultrasonic cavitation}

Ultrasonic cavitation is the phenomenon of breaking and immediate reconstruction of a fluid under the action of sufficiently high stress or rapid and sudden pressure variations.

Under the action of pressure changes, the mean distance between the liquid particles varies until the liquid breakdown value is reached. Breaking occurs when the ultrasonic pressure is negative and cancels the pressure corresponding to the cohesive forces between the liquid particles. Breaking is followed by genesis in those places of microscopic cavities. It does not occur simultaneously in the whole mass of the liquid, but only in weak points of resistance, generated by inhomogeneities called germs or cavitation nuclei. With the help of ultra-fast shooting, it was able to track and study the behavior of cavitation bubbles. Thus, it has been found that under the action of ultrasonic waves the cavitation bubble increases its volume by filling it with evaporative air or vapors that were present at the time of their formation. The cavity bubble develops to a certain size, which at a certain pressure depends on the duration of development and the frequency of ultrasounds. In the immediate next phase, after the relatively slow expansion of the cavitation bubble, sudden compression and sudden destruction occur. The duration $t$, necessary for the development of the spherical cavitation bubble, from the radius $\mathrm{R}_{0}$, to the radius $\mathrm{R}$, is given by the relation:

$$
t=\frac{3}{2} \rho R_{0} \int_{\frac{R_{0}}{R}}^{1} \frac{d\left(\frac{R_{0}}{R}\right)}{\left(\frac{R_{0}}{R}\right)^{2} \sqrt{\left(1-\frac{R_{0}}{R}\right)\left[\left(\left(\frac{R_{0}}{R}\right)^{2}+\frac{R_{0}}{R}\right)\left(p R_{0}+3 \sigma\right)\right]-p R_{0}}}
$$

where: $\rho$ is the density of the liquid; $p$ - hydrostatic pressure; $\sigma$ - surface stress of the liquid. 
In the immediate next phase, after the relatively slow expansion of the cavitation bubble, sudden compression and rapid destruction occur. The duration $\mathrm{t}$, required to compress the $\mathrm{R}_{\mathrm{m}}$ beam bubble at a radius $\mathrm{R}$, can be calculated with the relation:

$$
\tau=\sqrt{R_{m} \frac{3 \rho}{2 p}} \int_{\frac{R}{R_{m}}}^{1} \frac{\left(\frac{R}{R_{m}}\right)^{3 / 2} d\left(\frac{R}{R_{m}}\right)}{\sqrt{\left(1-\frac{R}{R_{m}}\right)\left[\left(\frac{R}{R_{m}}\right)^{2}+\left(\frac{R}{R_{m}}+1\right)\left(1+\frac{3 \sigma}{p R_{m}}\right)\right]}}
$$

As a result of the destruction of the cavitation bubble, the gas or vapor therein is compressed adiabatically, the temperature may reach up to $4 \times 10^{4}$ from the ambient temperature, approximately $40000^{\circ} \mathrm{C}$, and form a shock wave whose intensity increases as the ultrasonic pressure increases. Ultrasonic cavitation is the underlying phenomenon of many technical applications related to dimensional processing, filtration, cleaning, degreasing, deburring in the ultrasonic field, and therefore has been the subject of numerous researches. The phenomenon of cavitation is a dynamic process of forming, developing and breaking down some bubbles or cavities filled with vapors and gases in the mass of a liquid.

\subsection{Moderately clean, heterogeneous nucleation in liquids with solid particles in suspension}

It is admitted that microscopic particles suspended in a liquid, maintained in this state by Brownian motion, can serve as cavitation germs, as nuclei where liquid breakage may occur. It is postulated that a spherical solid sphere of radius $\mathrm{R}_{0}$ a vaporous cavity is formed with a spherical shape of radius $\mathrm{R}$. Thus, in the stable phase, solid core of radius $\mathrm{R}_{0}$ and volume $\mathrm{V}_{0}$ floats in the liquid with the volume $V+V_{v}$ and pressure $\mathrm{p}_{1}$ (Figure 1.a). In the vapor phase, volume $V_{v}$, pressure $p_{v}$ and radius $R$, surrounding the spherical particle, the $\mathrm{A}_{\mathrm{vl}}$ liquid-vapor separation surfaces are distinguished; $\mathrm{A}_{\mathrm{sp}}$ for the liquid-solid and the respective surface stress $\sigma_{v l}$ and $\sigma_{s l}$ (Figure 1.b).

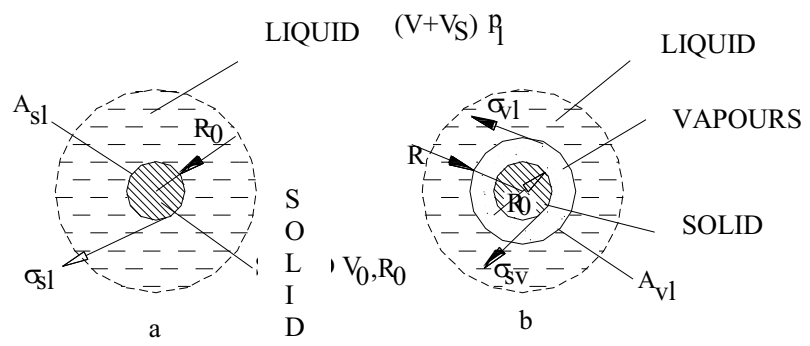

Fig. 1. The genesis of cavitation germ around the solid particle: $a-$ in the stable phase; $b$ - in the vapor phase.

This type of nucleation also favors the situation of ultrasonic air filtration, presented in the article. In this case, the thermodynamic potentials $\Omega_{1}$ and $\Omega_{2}$ become:

$$
\Omega_{1}=-p_{l}\left(V+V_{v}\right)-p_{01} V_{0}+\sigma_{s l} A_{s l}
$$




$$
\Omega_{2}=-p_{l} V-p_{v} V_{v}-p_{02} V_{0}+\sigma_{v l} A_{v l}+\sigma_{s v} A_{s v}
$$

Because:

$$
p_{01}=p_{1}+2 \frac{\sigma_{s l}}{R_{0}} ; p_{v}=p_{l}+2 \frac{\sigma_{l v}}{R} ; p_{o 2}=p_{l}+2 \frac{\sigma_{l v}}{R}+2 \frac{\sigma_{s v}}{R_{0}}
$$

and:

$$
V_{v}+V_{0}=\frac{4 \pi R^{3}}{3} ; A_{0}=4 \pi R_{0}^{2}
$$

It results:

$$
-\Delta F=\sigma_{l v}\left[\frac{2}{R}\left(V_{v}+V_{0}\right)-A_{l v}\right]-\sigma_{s l}\left[\frac{2}{R_{0}} V_{0}-A_{0}\right]+\sigma_{s v}\left[2 \frac{V_{0}}{R_{0}}-A_{0}\right]
$$

or:

$$
-\Delta F=-\frac{4 \pi R^{2}}{3} \sigma_{l v}-\frac{4 \pi R_{0}^{2}}{3}\left(\sigma_{s v}-\sigma_{s l}\right)
$$

By introducing the contact angle $\theta$, (Figure 2) given by the relation:

$$
\cos \theta=\frac{\sigma_{s v}-\sigma_{s l}}{\sigma_{l v}}
$$

\section{VAPOURS}

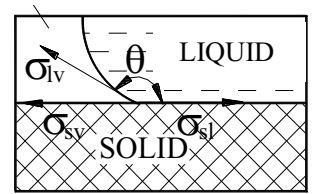

LIQUID

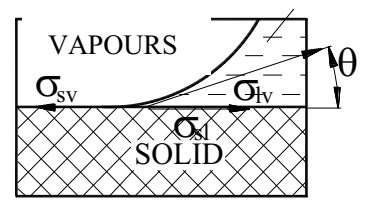

Fig. 2. Contact angle $\theta$, at the solid-liquid interface

we obtain the probability $\mathrm{P}$ of the occurrence of a cavitational nucleus around a solid particle in suspension, given by the relationship:

$$
P=e^{\frac{\Delta F}{k T}}=e^{\frac{4 \pi R^{2}}{3 k T} \sigma_{l}\left(1+\frac{R_{0}}{R} \cos \theta\right)}
$$

The variation of the probability $\mathrm{P}$ with the ratio $\frac{R}{R_{0}}$, in water at $27^{\circ} \mathrm{C}$, is presented in Figure 3.

It is seen that a particle of radius $R_{0}=10^{-6} \mathrm{~cm}$ being in suspension has an appropriate probability of remaining in this state, decreasing to about $P=e^{-20}$ when $R_{0}=5 \times 10^{-6} \mathrm{~cm}$. 


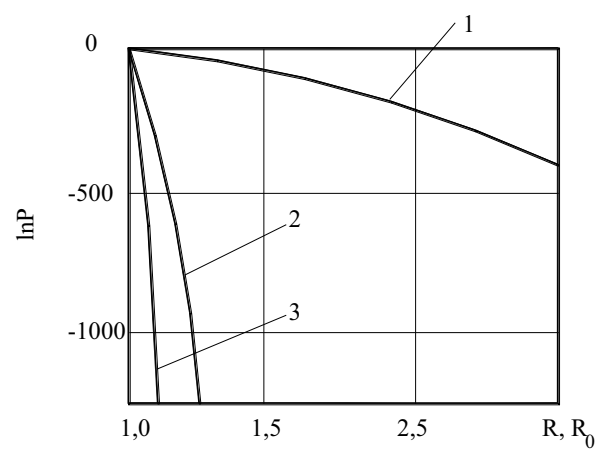

Fig. 3. Probability of occurrence of cavitation germ around the solid particle: $1-R_{0}=5 \times 10^{-7} \mathrm{~cm}$;

$2-R_{0}=5 \times 10^{-7} \mathrm{~cm} ; 3-R_{0}=10^{-6} \mathrm{~cm}$

It can be seen that around the frequency $\mathrm{f}=30 \mathrm{KHz}$, the phenomenon is relatively stable, after which the amplitude of the ultrasonic "noise" drops rapidly. Under these conditions, the design of an ultrasonic system in this frequency range is optimal.

In conclusion, it can be said that the filtration processing ultrasonic field is the result of the occurrence of the ultra-acoustic cavitation phenomenon and its acceleration due to the presence in the liquid medium of some cavitational germs introduced with the gaseous medium to be filtered and purified (all existing pollutants in the gaseous stream either solid, liquid or gaseous). This is also explained by the very high percentages of atmospheric pollutants (acetone, toluene, butylic acid, some hydrocarbons, etc.) because the ultraacoustic cavitation phenomenon accelerates the chemical dissolution of gases in the water and makes it possible to carry out chemical reactions which would not be possible without the action of ultrasonic energy.

\section{Calculation and dimensioning of the ultra-accoustic system used to obtain the ultra-acoustic cavitation phenomenon.}

In order to calculate and make the dimensioning of the ultrasonic system, the initial data necessary to solve the equations describing the vibrational behavior of the piezoceramic materials are first introduced [1,2].

- Material permissiveness - $\varepsilon_{0}=8.85 \times 10^{-12} \quad F / m \mathrm{j}=\sqrt{-1}$

- resonance frequency - $\mathrm{f}_{0}=3.5 \times 10^{4} \mathrm{~Hz}$

- resonance pulse - $\omega_{0}=2 \pi f_{0}$

- the minimum amplitude at the edge of the active part $-\xi=35 \times 10-6 \mathrm{~m}$.

- input electrical power $-\mathrm{P}_{\text {in }}=1000 \mathrm{~W}$

- minimum acoustic intensity $-\mathrm{I}_{\mathrm{a}}=160 \mathrm{~W} / \mathrm{cm}^{2}$

- acoustic-mechanical efficiency - $\eta=0.75$

- electromechanical coupling factor $-\zeta=0.65$

- electroacoustic efficiency $-\varepsilon_{\text {ea }}=0.98$

- density - $\rho=7.6 \cdot 10^{3} \mathrm{Kg} / \mathrm{m}^{3}$

- Young's module $-Y=7.6 \cdot 10^{10} \mathrm{~N} / \mathrm{m}^{2}$

- permittivity relative to $1 \mathrm{~Hz}-\varepsilon_{r p}=2600$

- angle of loss - $\delta_{\mathrm{p}}=0.6982 \mathrm{deg} ; \operatorname{tg}\left(\delta_{p}\right)=0.014$

- piezoelectric constant $-k_{p}=665 \cdot 10^{-12} \mathrm{~m} / \mathrm{V}$ 
- temperature Curie - $\Theta=560.15 \mathrm{~K}$

The reflector is made of titanium alloy (Ti5AlV) with the following characteristics:

- Young's module $-Y=80.3 \mathrm{~N} / \mathrm{mm}^{2}$

- density - $\rho=4.43 \cdot 10^{3} \mathrm{Kg} / \mathrm{m}^{3}$

Using the input data presented above, the dimensions of the transducer consisting of a passive element (reflector) and an active element will be calculated, assuming the determination of the following parameters $[3,4]$ :

- ultrasound propagation velocity through components:

$$
\begin{gathered}
v_{p}=\sqrt{\frac{Y}{\rho_{p}}}=3.25 \times 10^{3} \mathrm{~m} / \mathrm{s} \\
v_{T i}=\sqrt{Y_{T i} / \rho_{T i}}=4.258 \cdot 10^{3} \mathrm{~m} / \mathrm{s} \\
v_{O L}=\sqrt{Y_{O L} / Q_{O L}}=5.25 \times 10^{3} \mathrm{~m} / \mathrm{s}
\end{gathered}
$$

- ultrasound wavelength:

$$
\begin{aligned}
\lambda_{p} & =\frac{v_{p}}{f_{0}}=938 \mathrm{~mm} \\
\lambda_{T I} & =\frac{v_{p T i}}{f_{0}}=121.66 \mathrm{~mm} \\
\lambda_{O l} & =\frac{v_{O l}}{f_{0}}=150 \mathrm{~mm}
\end{aligned}
$$

The dimensions of the components according to the longitudinal direction of propagation of the ultrasonic vibrations in 4 are:

$$
\begin{aligned}
& d_{o l}=\frac{\lambda_{o l}}{4}=37.5 \mathrm{~mm} \\
& d_{p}=\frac{\lambda_{p}}{4 \cdot 2}=23.45 \mathrm{~mm} \\
& d_{T i}=\frac{\lambda_{T i}}{4}=30.415 \mathrm{~mm}
\end{aligned}
$$

The radiation field of the active element must be correlated with the input power and the required acoustic intensity and is:

$$
\begin{aligned}
& A_{p}=\frac{P_{\text {in }}}{I_{a \cdot \eta_{e a}}}=6.92 \mathrm{~cm}^{2} \\
& A_{T i}=A_{p}=6.92 \mathrm{~cm}^{2} \\
& A_{O l}=A_{p}=\mathrm{cm}^{2}
\end{aligned}
$$

- Active element radius (for circular section):

$$
r_{p}=\sqrt{\frac{A_{p}}{\pi}} 14.8 \mathrm{~mm}
$$

- Elements of calculation for the ultrasonic energy concentrator: 
- the length of the concentrator is calculated with the relation:

$$
L=\frac{n \cdot c}{2 \cdot f_{0}} \sqrt{1+\left[\frac{\ln (n)}{\pi n}\right]}=75.2 \mathrm{~mm}
$$

where: $f_{0}=2.8 \cdot 10^{4} \quad H z ; \mathrm{n}=1 ; \mathrm{N}=3.5$;

-nodal points, $\mathrm{x}_{\mathrm{nod}}$, are calculated with the following:

$$
x_{n o d}=\frac{L}{n \pi} \operatorname{arctg}\left(\frac{\ln N}{n \pi}+n^{\prime} \pi\right)
$$

where: $\mathrm{n}=1 ; \mathrm{n}{ }^{\prime}=1,2,3$

-the first nodal point is $\mathrm{x}_{\text {nod } 1}=38.3 \mathrm{~mm}$

As a result of the presented calculations, Figure 4 presents the schematic of the ultrasonic transducer used in air filtration system.

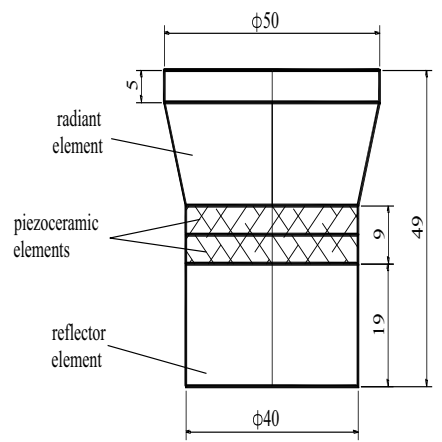

Fig. 4. Schematic of the piezoceramic transducer components used in ultrasonic air filtering system

\section{FEM modeling of the vibration modes of the transducers used in ultrasonic cavitation phenomenon}

The design of the ultrasonic active elements that are used to produce the ultrasonic cavitation phenomenon involves two important steps. The first is represented by the analytical calculation, after which, on the basis of the obtained data, the second step is the modeling and simulation of the ultrasonic system vibration modes [5, 6]. This second step is very useful because it offers a very complete and near-reality picture of how the ultrasonic transducer vibrates. From here it goes further and from the modes of vibration calculated and offered by the program will be chosen those that are useful for the proposed purpose. Characteristic of the useful vibration mode is the frequency at which it occurs so that using this frequency the ultrasonic generator can be tuned to provide optimal energy transfer and achieve the desired effect with minimal energy consumption.

In the first step of transducer modeling, the geometric shape presented in Figure 5was made. Since the piezoelectric phenomenon is characterized by the transformation of electric energy into mechanical energy, the next input data in the problem is represented by applying an electrical voltage on the surfaces of the piezoceramic elements. 


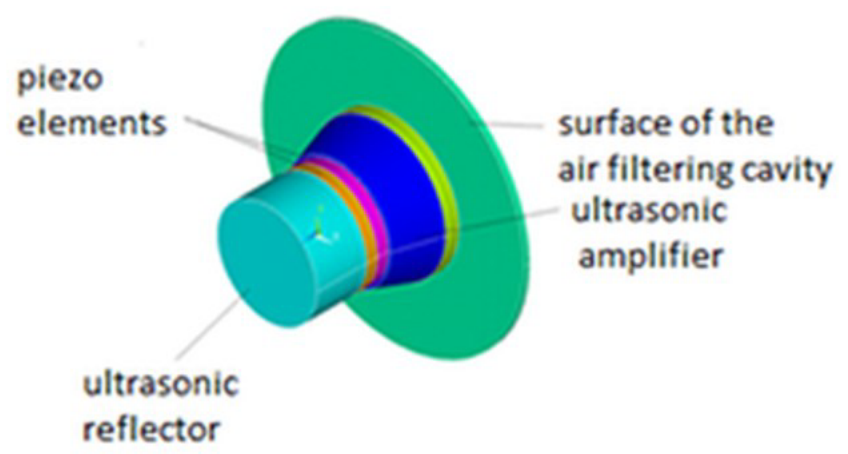

Fig. 5. Component elements and geometry of the piezoceramic transducer

Thus, the voltage $\mathrm{U}=200 \mathrm{~V}$ (the voltage value depends on the thickness of the piezoceramic transducers) is applied on their common surface, while on the other surfaces of the two piezoceramic elements the voltage $\mathrm{U}=0 \mathrm{~V}$ is applied. The surface of the tank was considered as having vibrate predominantly in the direction of OZ. For modal analysis a frequency range $\mathrm{f}=20 \mathrm{Khz} \ldots .100 \mathrm{Khz}$ was selected, the program looking for its own vibration modes under these conditions [7].

The third calculated vibration mode is produced at the frequency $\mathrm{f}=22419.3 \mathrm{~Hz}$. As can be seen, on the underside of the tank there are oscillations of the traveling wave, oscillations which can initiate very well the acoustic cavitation process by producing compressions and successive tensile stress of the liquid medium. The advantage of this vibrational mode is that, in addition to vibration, the piezoceramic elements do not deform in an undesirable way, with vibratory energy concentrating only on the surface of the ultrasonic bath. At the frequency $\mathrm{f}=22419.9 \mathrm{~Hz}$, very close to the previous one there is a vibration mode also very close to the previous one, the comment is similar (Figure 6).

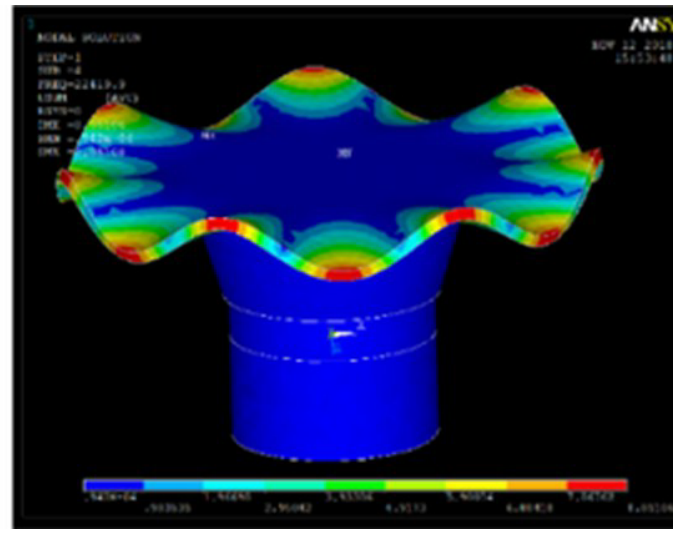

Fig. 6. Vibration mode at frequency $f=22419.9 \mathrm{~Hz}$.

The second mode of vibration occurs at the frequency $\mathrm{f}=26232 \mathrm{~Hz}$ and consists only in oscillations in the direction of the OZ. The oscillations are useful, the second advantage consisting in the absence of undesirable vibrations in the piezoceramic plates that do not deform by bending to the longitudinal axis. All vibrational energy is concentrated at the liquid level (Figure 7.a). At frequency $27319 \mathrm{~Hz}$, the vibration mode changes, it becomes similar to the first two modes presented, the advantage of which is that the piezoceramic disks do not deform unwanted, the possibility of their destruction being very small, therefore having a high durability (Figure 7.b). 


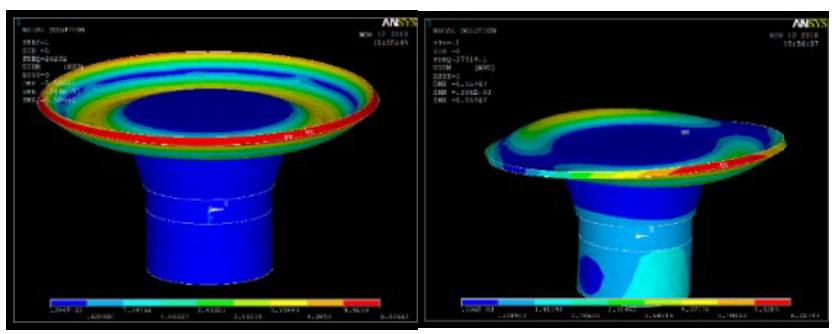

Fig. 7. Vibration mode at frequency: a - $f=26232 \mathrm{~Hz}$; b. - $f=27319 \mathrm{~Hz}$.

\section{Conclusions}

Article presents the steps in designing the main building elements used in the construction of an air filter operating on the principle of the ultra-acoustic cavity. In the first part there are presented some of the theoretical elements underlying the formation of cavitation bubbles, a phenomenon on which this type of filter is based. In the second part are presented some of the analytical elements that were studied and then solved so that it is possible to realize the piezoceramic transducer used in the production of the ultra-acoustic cavitation phenomenon. In the third part of the paper is presented the analysis of the main modes of vibration, the analysis done by the finite element method. This study is very useful as it allows the determination of the working frequencies of the system, which would otherwise have been achieved only through long experiments and probably without the best results. In this way, it is possible to optimize the filter function for as long as the only restriction in this respect is given by the piezoceramic elements working time.

This work has been funded by University POLITEHNICA of Bucharest, through the " PNCDI III, SP 2.1, CI" Identifier: NR. 189CI/2018 - "Installation for air preparation using ultrasonic filter system"

\section{References}

1. K. Nakamura, Ultrasonic transducers: Material and design for sensors, actuators and medical applications (Woodhead Publishing, 2012)

2. J. David, N. Cheeke, Fundamentals and applications of ultrasonic waves (CRC Press, 2012)

3. T.R. Gururaja, W.A. Schulze, L.E. Cross, R.E. Newnham, IEEE Transactions on Sonics and ultrasonics 32, 4 (1985)

4. C. Clésia, M. Korn, C. S. Sousa, A. Z. Arruda, J. Braz. Chem. Soc. 12, 1 (2001)

5. A. Safari, E. Koray Akdogan, Piezoelectric and Acoustic material for Transducer Applications (Springer, 2008)

6. K. Kikuchi, Ultrasounds: Their Application in Science and Technology (Springer, New York, 2009)

7. C. Amza, D. Niţoi, O. Chivu, C. Radu, M. Teodorescu, 15th International Multidisciplinary Scientific Geoconference (SGEM) (Albena, Bulgaria, 2015) 